\title{
Nonlinear Spiral Waves in a Galactic Disk
}

\author{
P. S. Alekseev and A. A. Danilenko \\ Ioffe Physicotechnical Institute, Russian Academy of Sciences, Politekhnicheskaya ul. 26, St. Petersburg, 194021 Russia \\ e-mail: alekseev_p_s@mail.ru \\ Received January 21, 2009
}

\begin{abstract}
An analytical solution of the nonlinear dynamic equations for a galactic disk is found. This solution describes the spiral design of spiral galaxies. The logarithmic profile of the spiral design and relatively larger density fluctuations as compared to velocity-field fluctuations are explained. Breaking of the obtained solution takes place for a certain critical amplitude. This can be a mechanism for the formation of galactic shocks and narrow star formation regions.
\end{abstract}

PACS numbers: 98.52.Nr, 98.56. Ne

DOI: $10.1134 / \mathrm{S} 0021364009050014$

1. The hypothesis of density waves was proposed in [1] to explain the spiral design of spiral galaxies. Spiral density waves in the linear approximation were constructed in [2] for a galactic disk described by hydrodynamic or collisionless kinetic equations. Serious difficulties are inherent in the model proposed in [2], which remained topical until recently. First, it was shown in [3] that the solutions derived in [2] are unstable in a wide range of the parameters describing models of galaxies. Second, in contrast to the assumption of the linear model [2], the difference between the inand off-arm densities is not small for actual galaxies [4]. Third, recent observations imply that many galaxies include dark-matter halos whose masses are, in many cases, much greater than the observed total mass of stars and gas [5]. This fact should also be taken into account at present in any description of galactic spiral designs. Finally, it is worthy to note that stars in spiral galaxies are most actively formed in increased density regions (in the spiral arms) [6]. However, these regions of active star formation are too narrow to be directly related to spiral waves [2]. In this respect, the possibility of existing large-scale shocks and corresponding narrow star formation regions related to spiral density waves was discussed in [7].

At the same time, we point to work [8] (see also [4, 9]) as the first nonlinear analysis of the spiral design performed in order to overcome the difficulties of the linear theory. Spiral density waves were treated as envelope solitons of small-scale perturbations. However, the soliton revealed in [8] was radially local and not azimuthally periodic.

In this work, an analytical solution of the hydrodynamic equations for the galactic disk is constructed in the form of nonlinear spiral waves in a certain disk annulus where variation in the azimuthally averaged angular velocity of rotation is negligible. It is assumed that the gravitational force is axially symmetric and caused by symmetrically distributed dark matter and/or radiating matter in the central part of the galaxy. The obtained solution describes the spiral design not only in a small part of the disk, as was done in [8], but in the entire annulus considered.

2. Following [2,6], we describe the dynamics of the gas component of the galactic disk by the following system of two-dimensional hydrodynamic equations:

$$
\begin{gathered}
\frac{\partial v_{r}}{\partial t}+v_{r} \frac{\partial v_{r}}{\partial r}+\frac{V_{\varphi}}{r} \frac{\partial V_{r}}{\partial \varphi}-\frac{V_{\varphi}^{2}}{r}=-\frac{\partial \Phi}{\partial r}, \\
\frac{\partial v_{\varphi}}{\partial t}+v_{r} \frac{\partial v_{\varphi}}{\partial r}+\frac{V_{\varphi}}{r} \frac{\partial V_{\varphi}}{\partial \varphi}+\frac{V_{r} V_{\varphi}}{r}=-\frac{1}{r} \frac{\partial \Phi}{\partial \varphi}, \\
\frac{1}{\sigma} \frac{\partial \sigma}{\partial t}+v_{r} \frac{1}{\sigma} \frac{\partial \sigma}{\partial r}+\frac{V_{\varphi}}{r} \frac{1}{\sigma} \frac{\partial \sigma}{\partial \varphi} \\
+\frac{1}{r} \frac{\partial\left(r v_{r}\right)}{\partial r}+\frac{1}{r} \frac{\partial V_{\varphi}}{\partial \varphi}=0,
\end{gathered}
$$

where $\Phi$ is the gravitational potential and $\sigma$ is the twodimensional gas density. The gas-kinetic pressure is neglected in Eqs. (1); i.e., the speed of sound is assumed to be much lower than the typical velocities $v_{r}$ and $v_{\varphi}$. First, such a description refers to the initial stage of galaxy evolution when the number of stars is still small. Second, it was shown in [10] that this approach is applicable even when stars are the major part of the galaxy matter.

Consider the galactic-disk annulus $r_{1}<r<r_{2}, 0<$ $\varphi<2 \pi$, where the angular velocity $\Omega_{0}$ averaged over the angle $\varphi$ can be assumed constant. This approximation holds at least for some galaxies (see Fig. 1 and review [4]). Under the assumption that the angular velocity is strictly independent of the variables $\varphi$ and $r$, the gravitational potential has the form

$$
\nabla \Phi=\alpha r \mathbf{e}_{r}, \quad \sqrt{\alpha}=\Omega_{0} .
$$



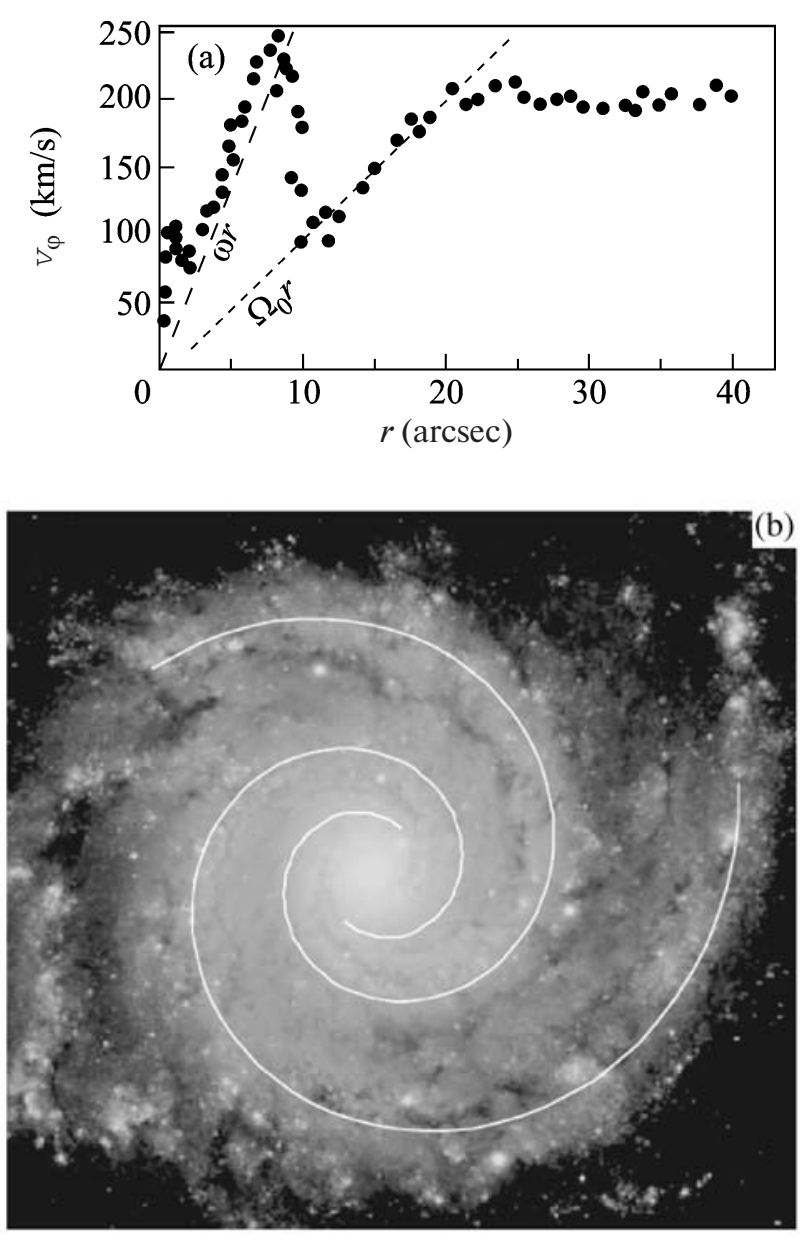

Fig. 1. (a) Rotation curve of the galaxy Mrk 1040. The figure is taken from review [4]. Two regions of constant angular velocity are clearly seen. The tentative "spiral-structure rotation curve" $v_{\text {spiral }}=\omega r$ is plotted on the basis of the estimate for the corotation-circle location [4] to illustrate the ratio between $\omega$ and $\Omega_{0}$ outside the corotation circle.

(b) The galaxy M74 and the logarithmic spirals $r=r_{0} e^{m \varphi / q}$, $r_{0} e^{m(\varphi+\pi) / q}$ with appropriately chosen parameters $(m / q \approx$ 0.2 ) superimposed onto its arms.

Correspondingly, we assume that there is axisymmetric gravitational potential (2) and seek a nonaxisymmetric solution of system (1) describing the spiral design. To be more correct, the deviation of the gravitational potential from Eq. (2) is assumed to be so small that its effects on the velocity and density fields are small in comparison with the typical field values in our solution based on the symmetric potential. In particular, the effect of the asymmetry of the gravitational potential on the asymmetry of the density field is small in comparison with the asymmetry of the density field for the constructed solution. A symmetric potential of the form given by Eq. (2) can be generated by the central stellar-gaseous part of the galaxy (this is similar to the approach [10]) and/or the spherical dark-matter halo [5].
The model disregarding the self-gravity is applicable due to the following estimates. The Poisson equation leads to relation between the gravity force $(\nabla \Phi)_{\text {gas }}$ caused by the gas and the two-dimensional gas density $\sigma_{\mathrm{gas}}(\mathbf{r})$ :

$$
(\nabla \Phi)_{\text {gas }}(\mathbf{r})=\mathbf{r} \int \frac{G \sigma_{\mathrm{gas}}\left(\mathbf{r}^{\prime}\right)}{\left|\mathbf{r}-\mathbf{r}^{\prime}\right|^{3}} d^{2} \mathbf{r}^{\prime} .
$$

The ratio $\delta$ of this force to, e.g., the term $v_{r}\left(\partial v_{r} / \partial r\right)$ in Eq. (1), is estimated as

$$
\delta \sim G \sigma_{\text {gas }} / v_{r}^{2} k,
$$

where $v_{r}$ and $k$ are the typical radial velocity and wavenumber of the velocity field in the constructed soliton. If, in contrast to [2, 10], loosely wounded spirals are considered (see Fig. 1) when the spiral design has only one or two turns, then $k$ is estimated as $1 / R$, where $R$ is the typical radius of the considered region of the galaxy. In this case, using the relation $G \rho_{\text {dark }} \sim \Omega_{0}^{2} \sim$ $V_{\varphi}^{2} / R^{2}$ between the rotation velocity $V_{\varphi}$ and the threedimensional density $\rho_{\text {dark }}$ of the dark matter, we have

$$
\delta \sim \frac{\sigma_{\text {gas }} / R}{\rho_{\text {dark }}} \frac{V_{\varphi}^{2}}{V_{r}^{2}} .
$$

Hence, if the ratio of the mass of the gas in the considered annulus to the dark-matter mass is small and the velocity perturbations with respect to the solid-rotation velocity in Eq. (1) are large (nonlinear), the anisotropic contribution from the self-gravity of the gas is negligible.

We seek the solution of system (1) in the form

$$
\begin{aligned}
& V_{r, \varphi}(r, \varphi, t)=\bar{V}_{r, \varphi}(r) \theta_{r, \varphi}(\psi), \\
& \sigma(r, \varphi, t)=\sigma_{0}(r) \exp \left[\theta_{\sigma}(\psi)\right],
\end{aligned}
$$

where $\psi=-\omega t+m \varphi+\phi(r)$ and $\theta_{i}$ are dimensionless $2 \pi$-periodic functions. Such an ansatz corresponds to only one assumption that the spiral-wave shape in the variable $\varphi$ is independent of the radius $r$. Representation (3) is similar to the general concept of the spiral design $[2,9,10]$, but we do not assume the trigonometric form of the functions $\theta_{i}$.

If the functions $\bar{V}_{i}, \sigma_{0}$, and $\phi$ are such that ansatz (3) is the solution of Eqs. (1), then it is reasonable to expect that representation (3) as a function of $\varphi$ and $t$ specifies the simplest periodic soliton of nonlinear system (1) for any $r$. Indeed, single-soliton solutions of classical nonlinear equations have the self-similar form, i.e., the wave quantity is a function of $\psi=x-v t$. This is also most probably valid for periodic solitons; e.g., this is the case for periodic solitons of the Korteweg-de Vries equation [11]. In [8], a function of the variables $r, \varphi$, and $t$ that is similar to that given by Eqs. (3) and is a solution of the dynamic equations for the gas disk in the cylindrical coordinates is also called soliton. 
The simplest solution given by Eqs. (3) is a solid rotation $v_{\varphi}=\Omega_{0} r$ and $v_{r}=0$. Ansatz (3) also includes the linear regime. Indeed, substituting the following velocity field into Eqs. (1):

$$
\begin{gathered}
V_{\varphi}(r, \varphi, t)=\Omega_{0} r+a(r) e^{i(-\omega t+m \varphi)}, \\
V_{r}(r, \varphi, t)=b(r) e^{i(-\omega t+m \varphi)}, \\
a(r), b(r) \ll \Omega_{0} r,
\end{gathered}
$$

we find that the functions $a(r)$ and $b(r)$ obey the following system of equations (the terms with derivatives with respect to $r$ are fortunately cancelled):

$$
\begin{gathered}
-2 \Omega_{0} a+i\left(-\omega+m \Omega_{0}\right) b=0, \\
i\left(-\omega+m \Omega_{0}\right) a+2 \Omega_{0} b=0 .
\end{gathered}
$$

Equations (4) imply that small perturbations in this case are not plane waves, but can be arbitrary functions of $r$. This means that it would be better not to apply such a model in the linear approximation, because allowance for self-gravity and dissipation would inevitably affect the dependence of perturbations on $r$. However, Eqs. (4) lead to a relation between the amplitudes and phases of the functions $a$ and $b$ and to the following dispersion relation specifying the rotation frequency $\omega$ of the spiral design ${ }^{1}$ :

$$
\tilde{\omega} \equiv \omega / \Omega_{0}=m-2 \text {. }
$$

3. The substitution of Eqs. (3) into Eqs. (1) shows that Eqs. (3) specify the solution if, and only if,

$$
\begin{gathered}
\bar{V}_{r, \varphi}(r)=\Omega_{0} r, \quad \phi(r)=q \ln \left(r / r_{0}\right), \\
\sigma_{0}(r)=\mu r^{\gamma},
\end{gathered}
$$

where $q, r_{0}, \mu$, and $\gamma$ are arbitrary parameters. If Eqs. (6) are satisfied, then we arrive at the following system of two ordinary first-order differential equations for the functions $\theta_{r}(\psi)$ and $\theta_{\varphi}(\psi)$ :

$$
\begin{aligned}
& \frac{d \theta_{r}}{d \psi}=\frac{\theta_{r}^{2}-\theta_{\varphi}^{2}+1}{-\tilde{\omega}+q \theta_{r}+m \theta_{\varphi}}, \\
& \frac{d \theta_{\varphi}}{d \psi}=\frac{2 \theta_{r} \theta_{\varphi}}{-\tilde{\omega}+q \theta_{r}+m \theta_{\varphi}} .
\end{aligned}
$$

The periodicity of the functions $\theta_{i}(\psi)$ is ensured by the boundary conditions $\theta_{i}(0)=\theta_{i}(2 \pi)$. By solving the boundary value problem formulated above for $\theta_{r}(\psi)$ and $\theta_{\varphi}(\psi)$ in the interval $0<\psi<2 \pi$ and taking into account the discussion in Section 2, we thereby construct a periodic one-soliton solution of system (1) for fixed $r$. Note that the existence of waves given by Eqs. (3) with the phase and amplitude given by Eqs. (6) with an arbitrarily strong nonlinearity is a

\footnotetext{
${ }^{1}$ Note also the existence of the solution $\tilde{\omega}=m+2$ corresponding to a decreasing function $\psi(\chi)$ (in particular, $\psi(2 \pi)=-2 \pi$ ) in the nonlinear analysis developed in Section 3. The characteristics of the resulting solitons for $\tilde{\omega}_{ \pm}=m \pm 2$ are similar.
}

remarkable fact related to the structure of the hydrodynamic operator $(\mathbf{v} \cdot \nabla) \mathbf{v}$.

The third of Eqs. (1) together with Eqs. (3) yields an explicit algebraic expression for $\theta_{\sigma}^{\prime}$ in terms of $\theta_{r}$ and $\theta_{\varphi}$. The periodicity condition for the function $\theta_{\sigma}(\psi)$ yields $\gamma=-2$. As a result,

$$
\exp \left[\theta_{\sigma}(\psi)\right]=\frac{1}{-\tilde{\omega}+q \theta_{r}(\psi)+m \theta_{\varphi}(\psi)} .
$$

It is easy to obtain the first integral of system (7):

$$
\theta_{r}^{2}+\left(\theta_{\varphi}-C\right)^{2}=C^{2}-1
$$

It is seen that the phase trajectories of system (7) are embedded nonconcentric circles specified by the constant $C,|C| \geq 1$. The circle radii are $\lambda=\sqrt{C^{2}-1}$. The sign of the constant $C$ determines the resulting sign of $V_{\varphi}$, i.e., the direction of rotation. The point $(0,1)$ $(C=1)$ is also a solution and corresponds to solid rotation. The small circles correspond to the linear regime of small velocity perturbations. The increase in the radius corresponds to an increase in the soliton nonlinearity. Let us parameterize the circles in terms of the polar angle $\chi$ as follows:

$$
\theta_{r}=\lambda \cos \chi, \quad \theta_{\varphi}=\sqrt{\lambda^{2}+1}+\lambda \sin \chi .
$$

From the first of Eqs. (7), $\psi$ is expressed in terms of $\chi$ as

$$
\psi(\chi)=\int_{\theta_{r}(0)}^{\theta_{r}(\chi)} \frac{-\tilde{\omega}+q \theta_{r}+m \theta_{\varphi}}{\theta_{r}^{2}-\theta_{\varphi}^{2}+1} d \theta_{r} .
$$

Explicit formulas for the function $\psi(\chi)$ are obtained from Eq. (10) using Eqs. (9). Assuming that $\psi(0)=0$, we find $\psi(\chi)=\psi_{1}(\chi)$ for the angles $0<\chi<\pi$ and $\psi(\chi)=\psi_{1}(\chi)-\tilde{\omega} \pi$ for $\pi<\chi<2 \pi$, where

$$
\begin{aligned}
& \psi_{1}(\chi)=-\tilde{\omega} \arctan \left[\sqrt{\lambda^{2}+1} \tan (\chi / 2)+\lambda\right] \\
& +\tilde{\omega} \arctan (\lambda)+\frac{q}{2} \ln \left[\frac{\lambda \sin \chi}{\sqrt{\lambda^{2}+1}}+1\right]+\frac{m \chi}{2} .
\end{aligned}
$$

The boundary conditions $\theta_{r, \varphi}(0)=\theta_{r, \varphi}(2 \pi)$ are satisfied if $\psi(2 \pi)=2 \pi$. This requirement has the meaning of a "nonlinear dispersion relation" specifying the frequency $\tilde{\omega}$ in terms of the parameters $q, r_{0}, m$, and the constant $C$ specifying the circles. It is remarkable that, meanwhile, the requirement $\psi(2 \pi)=2 \pi$ yields the same dispersion relation given by Eq. (5) as in the linear case. (The constant $C$ does not appear in the nonlinear dispersion relation.) The quantities $q$ and $r_{0}$ remain arbitrary for any $m$ value. In particular, this means that neither leading nor trailing spirals are preferential in the developed theory.

Equation (11) together with Eq. (5) yields $\psi(\chi)=\chi$ for $\lambda \longrightarrow 0$. This corresponds to the linear regime. As the soliton amplitude increases, its profile becomes essentially anharmonic. The function $\chi(\psi)$ becomes 


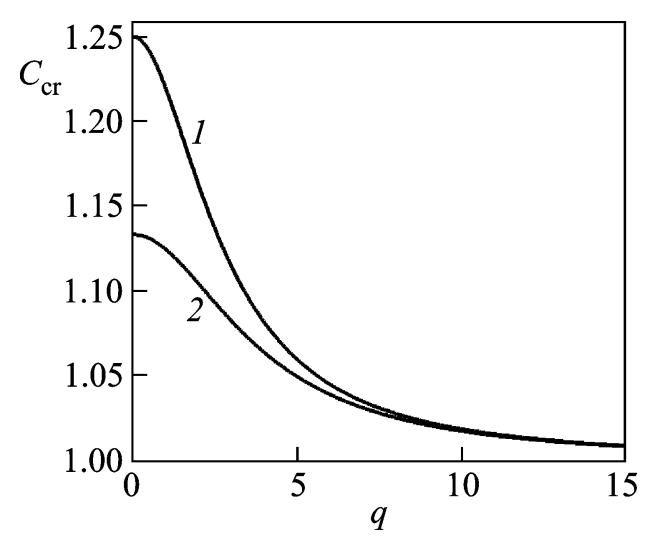

Fig. 2. Quantity $C_{\mathrm{cr}}$ versus the parameter $q$ for $m=$ (curve 1) -2 and (curve 2) -3 .

multivalued at some point $\psi_{\mathrm{cr}}$ for certain $C=C_{\mathrm{cr}}$. Hence, the functions $\theta_{i}(\psi)$ also become multivalued. This situation, called wave breaking in hydrodynamics, means that the accepted model fails in this case and dissipation must be taken into account. The broken front is smoothed and describes a shock if dissipation is taken into account. The quantities $C_{\mathrm{cr}}$ and $\chi_{\mathrm{cr}}=$ $\chi\left(\psi_{\mathrm{cr}}, C_{\mathrm{cr}}\right)$ are found from the system of equations

$$
\begin{aligned}
\psi^{\prime}(\chi, C) & =0, \\
\psi^{\prime \prime}(\chi, C) & =0 .
\end{aligned}
$$

Figure 2 shows $C_{\mathrm{cr}}$ as a function of $q$ for two $m$ values. A decrease in $C_{\mathrm{cr}}$ with increasing $q$ is fairly clear, since larger $q$ values correspond to more densely wounded spirals.

Figure 3 shows the functions $\theta_{\varphi}(\psi)$ and $\exp \left[\theta_{\sigma}(\psi)\right]$ for various values of the parameter $C$. The transition of the soliton from the linear regime to the essentially nonlinear regime with a variation in $C$ from 0 to $C_{\mathrm{cr}}$ is clearly seen. It is seen that if $C$ is sufficiently close to $C_{\mathrm{cr}}$, then fixed relative variations in $\theta_{\varphi}$ in the soliton correspond to much larger relative variations in the inand off-spiral densities. This is also explained by the following consideration. If fluctuations of $\theta_{\varphi}$ in the soliton are about $\theta_{\varphi}$, then, according to the algebraic relation between $\theta_{r}, \theta_{\varphi}$, and $\theta_{\sigma}^{\prime}$, fluctuations of $\theta_{\sigma}$ are about $\theta_{\sigma}$. In this case, fluctuations of $\sigma$ are much larger than the mean value of $\sigma$, since an exponential function enters into the second of Eqs. (3). It is also seen in Fig. 3 that the density becomes infinitely high if $(\psi, C)=(\psi, C)_{\mathrm{cr}}$. This follows from Eqs. (8) and (10) and the fact that the numerator in integral (10) is also zero if $\psi^{\prime}(\chi)=0$.

4. The above consideration shows that the spiral design is a specific type of nonlinear waves inherent in a gas disk in a symmetric external field. This disproves the conventional opinion that a lower symmetry generator is necessary to sustain the spiral design (bar [12] or satellite galaxy [13]). The logarithmic shape of spirals is predicted in agreement with observations (see
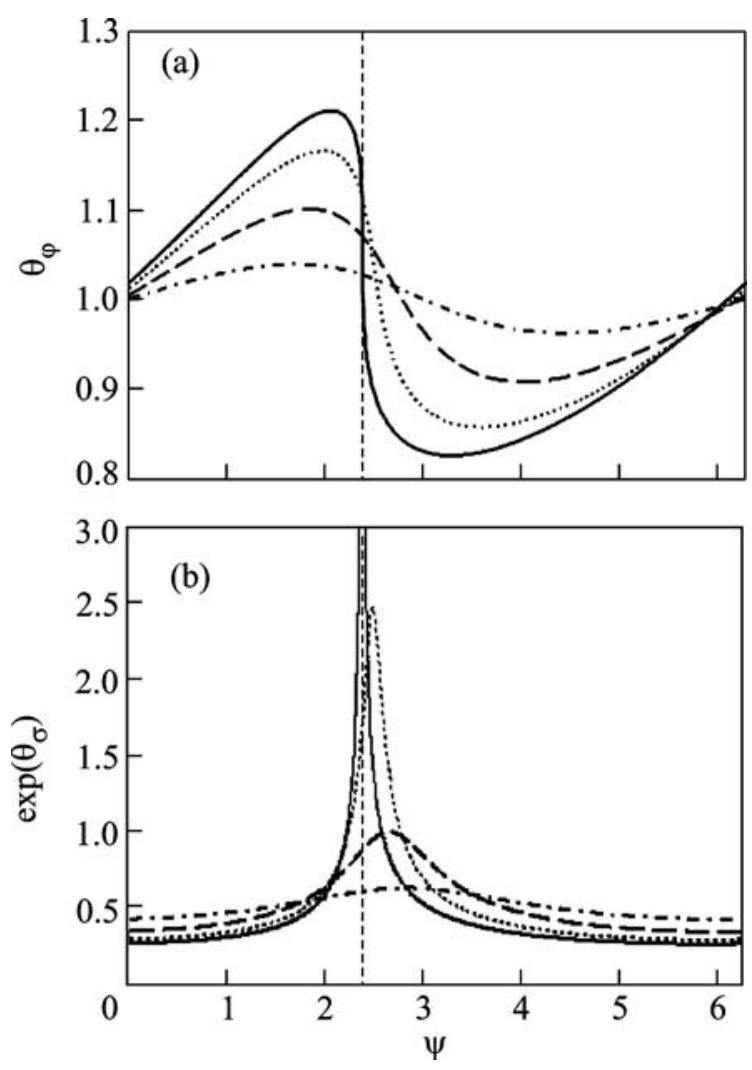

Fig. 3. Profiles of the soliton functions (a) $\theta_{\varphi}(\psi)$ and (b) $\exp \left[\theta_{\sigma}(\psi)\right]$ for the parameters $q=10$ and $m=-2$ and the soliton amplitude $\lambda=$ (dash-dotted curve) 0.2 , (dashed curve) 0.5 , (dotted curve) 0.8 , and (solid curve) 1.0 versus its maximum possible value $\lambda_{\mathrm{cr}}=\sqrt{C_{\mathrm{cr}}^{2}-1}$. The vertical dashed line marks the point $\psi_{\mathrm{cr}}$.

Fig. 1 in [13]). The angular velocity of the spiral design is related to the number of arms and gas angular velocity by formula (5). For example, $|\omega|=4 \Omega_{0}$ for $m=-2$, which is fairly reasonable taking into account Fig. 1. In this respect, this model seems to be the most appropriate model for galactic-disk regions outside the corotation circle. The presence of density fluctuations exceeding the velocity-field fluctuations, which is often observed in the spiral design [4], is explained. It is reasonable to associate the region with the sharp peak of the function $\theta_{\sigma}(\psi)$ for $C \longrightarrow C_{\mathrm{cr}}$ with a narrow star-formation region in the spiral arm. The constructed model implies the breaking of waves with sufficiently large amplitudes. This can provide a mechanism for the generation of galactic shocks. The stability of the derived solution is probably related to its soliton-like form in the sense of [8], but we think that this should be carefully checked.

We are sincerely grateful to D.P. Barsukov, D.A. Varshalovich, A.P. Dmitriev, D.A. Zyuzin, A.V. Ivanchik, V.Yu. Kachorovskii, S.G. Poltorak, E.V. Polyachenko, S.A. Rodionov, D.S. Svinkin, 
A.V. Khoperskov, D.G. Yakovlev, and I.N. Yassievich for numerous helpful discussions and to A.P. Alekseeva, A.Yu. Zyuzin, and A.A. Prokof'ev for reading the manuscript and whose advice improved it. This work was supported by the Russian Foundation for Basic Research (project no. 08-02-00837), the Council of the President of the Russian Federation for Support of Young Scientists and Leading Scientific Schools (project no. NSh-2600.2008.2), and the Dynasty Foundation.

\section{REFERENCES}

1. B. Lindblad and R. G. Langebartel, Stockholm Obs. Ann. 17, 6 (1953).

2. C. C. Lin and F. H. Shu, Astrophys. J. 140, L646 (1964); C. C. Lin, C. Yuan, and F. H. Shu, Astrophys. J. 155, L721 (1969).

3. A. Toomre, Astrophys. J. 158, 899T (1969).

4. A. M. Fridman, Usp. Fiz. Nauk 177, 121 (2007) [Phys. Usp. 50, 115 (2007)].

5. M. Kamionkowski, arXiv:astro-ph/0706.2986 v1 (June 20, 2007); L. Bergstrom, Rep. Prog. Phys. 63, $793(2000)$.
6. Ya. B. Zeldovich and I. D. Novikov, The Structure and Evolution of the Universe (Nauka, Moscow, 1975; Univ. of Chicago, Chicago, 1983), pp. 386-449.

7. W. W. Roberts, Astrophys. J. 158, 123 (1969).

8. A. B. Mikhailovsky, V. I. Petviashvili, and A. M. Fridman, Pis'ma Zh. Eksp. Teor. Fiz. 26, 129 (1977) [JETP Lett. 26, 141 (1977)].

9. V. L. Polyachenko and A. M. Fridman, Equilibrium and Stability of Gravitating Systems (Nauka, Moscow, 1976), pp. 330-336, 365-410 [in Russian].

10. A. M. Fridman, Zh. Eksp. Teor. Fiz. 98, 1121 (1990) [Sov. Phys. JETP 71, 627 (1990)].

11. V. E. Zakharov, S. V. Manakov, S. P. Novikov, and L. P. Pitaevsky, Soliton Theory: Inverse Scattering Method (Nauka, Moscow, 1980; Plenum, New York, 1984), pp. 112-186.

12. K. Wada and C. A. Norman, Astrophys. J. 547, 172 (2001); K. Wada, G. Meurer, and C. A. Norman, Astrophys. J. 577, 197 (2002).

13. Yu. N. Efremov, "Stellar Complexes and Spiral Arms," Lecture on the School of Young Scientists on Galaxy Physics, SAO RAS, Sept. 2007.

Translated by A. Serber 